\title{
Influence of pretreated activated sludge for electricity generation in microbial fuel cell application.
}

\begin{abstract}
Influence of different pretreated sludge for electricity generation in microbial fuel cells (MFCs) was investigated in this study. Pre-treatment has shown significant improvement in MFC electricity productivity especially from microwave treated sludge. Higher COD reduction in the MFC has been revealed from microwave treated sludge with 55\% for total and $85 \%$ for soluble COD, respectively. Nonetheless, longer ozonation treatment did not give additional advantage compared to the raw sludge. On the other hand, samples from anodes were analyzed using the 16S rRNA gene-based pyrosequencing technique for microbial community analysis. There was substantial difference in community compositions among MFCs fed with different pretreated sludge. Bacteroidetes was the abundant bacterial phylum dominated in anodes of higher productivity MFCs. These results demonstrate that using waste sludge as the substrate in MFCs could achieve both sludge reduction and electricity generation, and proper pre-treatment of sludge could improve the overall process performance.
\end{abstract}

Keyword: Microbial fuel cell; Sludge; Ozonation; Microwave; Pyrosequencing. 